\title{
Advances in MRI Assessment of Gliomas and Response to Anti-VEGF Therapy
}

\author{
Whitney B. Pope • Jonathan R. Young • \\ Benjamin M. Ellingson
}

Published online: 15 January 2011

(C) The Author(s) 2011. This article is published with open access at Springerlink.com

\begin{abstract}
Bevacizumab is thought to normalize tumor vasculature and restore the blood-brain barrier, decreasing enhancement and peritumoral edema. Conventional measurements of tumor response rely upon dimensions of enhancing tumor. After bevacizumab treatment, glioblastomas are more prone to progress as nonenhancing tumor. The RANO (Response Assessment in Neuro-Oncology) criteria for glioma response use fluid-attenuated inversion recovery (FLAIR)/T2 hyperintensity as a surrogate for nonenhancing tumor; however, nonenhancing tumor can be difficult to differentiate from other causes of FLAIR/T2 hyperintensity (eg, radiation-induced gliosis). Due to these difficulties, recent efforts have been directed toward identifying new biomarkers that either predict treatment response or accurately measure response of both enhancing and nonenhancing tumor shortly after treatment initiation. This will allow for earlier treatment decisions, saving patients from the adverse effects of ineffective therapies while allowing them to try alternative therapies sooner. An active area of research is the use of physiologic imaging, which can potentially detect treatment effects before changes in tumor size are evident.
\end{abstract}

Keywords Gliomas · Glioblastoma · MR diffusion · MR perfusion $\cdot$ MR spectroscopy $\cdot$ Radiotracers $\cdot$ Angiogenesis inhibitors $\cdot$ Bevacizumab

\footnotetext{
W. B. Pope $(\bowtie) \cdot J$. R. Young $\cdot$ B. M. Ellingson

Department of Radiological Sciences, David Geffen School of Medicine at UCLA,

10833 Le Conte Avenue, BL-428 CHS,

Los Angeles, CA 90095-1721, USA

e-mail: wpope@mednet.ucla.edu
}

\section{Introduction}

Of the different types of glioma, glioblastoma (GBM) is the most common and aggressive, with a median survival of 12 to 15 months [1]. Multiple antiangiogenic drugs are currently in use or in clinical trials for the treatment of GBM. The prototype, bevacizumab, an antibody to vascular endothelial growth factor (VEGF), is now commonly used in the setting of recurrent GBM. However, only a fraction of patients with malignant gliomas respond to antiangiogenic therapy, and "response" in this setting is not well defined [1]. Thus, it is critically important to identify radiologic biomarkers that can either predict treatment response or accurately measure response after the initiation of therapy, thereby improving decision making and ultimately increase survival. The goals of this article are to review current criteria for glioma response and to discuss new MRI techniques that may provide potential biomarkers of treatment effect.

\section{Criteria for Assessing Glioma Response}

In the assessment of treatment response, MRI has traditionally been used to evaluate tumor size. Changes in enhancing tumor size based on bidimensional measurements are the basis for both the RANO (Response Assessment in Neuro-Oncology) criteria, the currently accepted standard criteria for assessing glioma response, and the previously used standard, the Macdonald criteria [2]. Although relying on conventional T1-weighted postcontrast scans worked well in the past in evaluating treatment response, the widespread adoption of bevacizumab as salvage chemotherapy has highlighted its limitations. The limitations stem largely from bevacizumab's 
antipermeability effect. GBMs typically have extensive abnormal vasculature with a blood-brain barrier that is significantly more permeable than normal brain tissue [3]. Because of the increased permeability, contrast material more freely leaks out of tumor capillaries, increasing enhancement on T1-weighted images. By targeting VEGF, an active permeability agent and promoter of angiogenesis, bevacizumab decreases the leak of contrast agent into the interstitium, diminishing contrast enhancement. Because of the effects of bevacizumab on tumor vasculature, a reduction in contrast enhancement may not necessarily reflect a cytotoxic or cytostatic tumor response. Relying on contrast enhancement can thus lead to an overestimation of treatment response, a phenomenon known as "pseudoresponse" [4, 5].

The impact of bevacizumab on radiation necrosis is another important consideration in assessing radiographic response. Radiation necrosis is typically associated with avid enhancement, edema, and potentially mass effect, making it difficult to distinguish from tumor. Because of the potential similarity with true tumor progression, radiation changes can lead to "pseudoprogression" [4, 5]. It is estimated that in $20 \%$ to $30 \%$ of patients with GBM, the first postradiation MRI shows an increase in contrast enhancement that subsides with time without any change in therapy, and may therefore represent radiation change, rather than true tumor progression [2, 5]. However, no careful studies have been performed that have determined whether these sometimes minor enhancements are truly confused with tumor progression by an experienced interpreter in a clinical setting. And it also is likely that ambiguities in interpretation can often be resolved on close interval follow-up. Thus, the $20 \%$ to $30 \%$ figure reported may be a substantial overestimation of clinically relevant "pseudoprogression." Regardless, the physiologies underlying tumoral necrosis and radiation necrosis are similar, as they both are associated with leaky vasculature and increased levels of VEGF [6]. This provides a mechanism whereby they respond similarly to bevacizumab: in the same way that bevacizumab can improve the radiographic appearance of necrotic tumor, it can also substantially improve the radiographic appearance of radiation necrosis by decreasing enhancement and edema [7]. It has been suggested that the responses seen in bevacizumab-treated patients may be due, at least in part, to palliation of radiation necrosis rather than true tumor response [7].

To address the limitations in the conventional MRI assessment of gliomas, the RANO group has developed new guidelines for treatment response in brain tumors [2]. In contrast to the Macdonald criteria, the RANO criteria (summarized in Table 1) include fluid-attenuated inversion recovery (FLAIR) or T2 hyperintensity as a surrogate for nonenhancing tumor in the determination of progression. Unfortunately, it does not quantify the amount of FLAIR or
Table 1 Summary of the RANO criteria

\begin{tabular}{|c|c|}
\hline Response & Criteria \\
\hline Complete response & $\begin{array}{l}\text { Disappearance of all enhancing measurable } \\
\text { and nonmeasurable disease sustained for a } \\
\text { minimum of } 4 \text { weeks } \\
\text { Stable or improved FLAIR/T2 lesions } \\
\text { No new lesions } \\
\text { Clinical status is stable or improved } \\
\text { Patients cannot be receiving corticosteroids } \\
\text { (physiologic replacement doses are acceptable) }\end{array}$ \\
\hline Partial response & $\begin{array}{l}50 \% \text { or greater decrease (compared with baseline) } \\
\text { in the sum of products of perpendicular } \\
\text { diameters of all measurable enhancing lesions } \\
\text { sustained for a minimum } \\
\text { of } 4 \text { weeks } \\
\text { No progression of nonmeasurable disease } \\
\text { No new lesions } \\
\text { Stable or improved FLAIR/T2 lesions } \\
\text { Clinical status is stable or improved } \\
\text { The corticosteroid dosage at the time of the } \\
\text { scan should be no greater than the dosage } \\
\text { at the time of the baseline scan }\end{array}$ \\
\hline Stable disease & $\begin{array}{l}\text { Patient does not qualify for complete } \\
\text { response, partial response, or progression } \\
\text { Stable FLAIR/T2 lesions on a corticosteroid } \\
\text { dose no greater than at baseline } \\
\text { Clinical status is stable }\end{array}$ \\
\hline Progression & $\begin{array}{l}\text { Defined by any of the following: } \\
25 \% \text { or greater increase in sum of the products } \\
\text { of perpendicular diameters of all measurable } \\
\text { enhancing lesions compared with the smallest } \\
\text { tumor measurement obtained either at } \\
\text { baseline or best response following the } \\
\text { initiation of therapy, while on a stable or } \\
\text { increasing dose of corticosteroids. } \\
\text { Significant increase in FLAIR/T2 lesions } \\
\text { compared with baseline or best response } \\
\text { following initiation of therapy, not caused by } \\
\text { comorbid events (eg, radiation therapy, } \\
\text { ischemic injury, seizures, postoperative } \\
\text { changes, or other treatment effects), while on } \\
\text { a stable or increasing dose of corticosteroids } \\
\text { Presence of new lesions } \\
\text { Clinical deterioration not attributable to other } \\
\text { causes apart from the tumor (eg, seizures, } \\
\text { medication side effects, complications of } \\
\text { therapy, cerebrovascular events, infection) } \\
\text { or decreases in corticosteroid dose } \\
\text { Failure to return for evaluation due to death } \\
\text { or deteriorating condition } \\
\text { Clear progression of nonmeasurable disease }\end{array}$ \\
\hline
\end{tabular}

FLAIR fluid-attenuated inversion recovery, RANO Response Assessment in Neuro-Oncology. (Adapted from Wen et al. [2]) 
T2 change required for progression. Due to difficulties in differentiating nonenhancing tumor from other causes of increased FLAIR or T2 signal (eg, radiation effects, ischemic injury, and infection), the RANO group felt that an objective criterion for nonenhancing tumor progression was not currently feasible. Thus, it is clear that methods separating nonenhancing tumor from gliosis, and true tumor progression from pseudoprogression, could substantially improve the accuracy of response measures.

\section{New Imaging Techniques and the Search for Biomarkers for Glioma Response}

\section{Magnetic Resonance Perfusion}

The use of perfusion imaging as a biomarker for response to antiangiogenic drugs has generated significant interest. There are no validated biomarkers for antiangiogenesis that are currently available for clinical use. The two most common methods of perfusion imaging are dynamic susceptibility contrast (DSC) imaging and dynamic contrast-enhanced
(DCE) imaging. DSC imaging can be used to generate maps of relative cerebral blood volume ( $\mathrm{rCBV}$ ), relative cerebral blood flow (rCBF), and mean transit time. Other DSC parameters include relative peak height, which is correlated with $\mathrm{rCBV}$, and percentage of signal intensity recovery (PSR), which is a marker for capillary permeability. DCE imaging is generally used to measure K-trans, a constant for the rate at which contrast material moves from the vasculature to the extracellular space. K-trans is dependent upon the surface area of capillaries, as well as the leakiness of the capillaries. Thus, $\mathrm{K}$-trans is often used as a marker for the permeability of tumor vasculature. Using these parameters, many groups have investigated the role of perfusion imaging in evaluating gliomas. Table 2 provides a summary of the key findings from recent magnetic resonance (MR) perfusion studies. Hirai et al. [8] studied 49 patients with high-grade astrocytomas prior to the initiation of radiation and chemotherapy and found that $\mathrm{rCBV}$ has prognostic value. Using a multivariate regression model to control for age and other clinical features, maximum rCBV was found to be an independent prognostic factor of survival: the 2-year survival rate was $67 \%$ for patients with a maximum rCBV below 2.3
Table 2 Key findings of MR perfusion studies in the assessment of gliomas
$D C E$ dynamic contrast enhanced, DSC dynamic susceptibility contrast, $H P V$ hyperperfusion volume, $M R$ magnetic resonance, $P S R$ percentage of signal intensity recovery, $r C B V$ relative cerebral blood volume, $r P H$ relative peak height

\begin{tabular}{|c|c|}
\hline Study & Key finding(s) \\
\hline \multicolumn{2}{|l|}{ DSC perfusion } \\
\hline \multicolumn{2}{|c|}{ Prognostic value for standard treatment (chemotherapy and radiation) } \\
\hline Hirai et al. [8] & $\begin{array}{l}\text { Maximum rCBV was prognostic of 2-year survival in patients with high-grade } \\
\text { astrocytomas }\end{array}$ \\
\hline Mangla et al. [14] & Change in $\mathrm{rCBV}$ was predictive of 1 -year survival in patients with glioblastoma \\
\hline Law et al. $[15 \bullet \bullet]$ & rCBV was predictive of time to progression in both high- and low-grade gliomas \\
\hline \multicolumn{2}{|c|}{ Predictive value for response to antiangiogenic therapy } \\
\hline Sawlani et al. [9] & Change in HPV correlated with time to progression in recurrent glioblastoma patients \\
\hline Akella et al. [13] & $\begin{array}{l}\text { rCBF was correlated with radiographic and clinical response in patients with malignant } \\
\text { gliomas treated with cilengitide }\end{array}$ \\
\hline \multicolumn{2}{|c|}{ Differentiating true progression from pseudoprogression } \\
\hline Barajas et al. $[18 \bullet \bullet]$ & $\begin{array}{l}\text { DSC parameters (rCBV, rPH, PSR) were significantly different in recurrent } \\
\text { glioblastoma compared with radiation necrosis }\end{array}$ \\
\hline Hu et al. [19] & $\begin{array}{l}\text { An rCBV threshold value of } 0.71 \text { differentiated high-grade glioma recurrence from } \\
\text { post-treatment radiation effect with a sensitivity of } 91.7 \% \text { and a specificity of } 100 \%\end{array}$ \\
\hline Sugahara et al. [20] & $\begin{array}{l}\text { rCBV ratios were }>2.6 \text { in cases of recurrent tumor and }<0.6 \text { in cases of post-treatment } \\
\text { radiation effect }\end{array}$ \\
\hline Gahramanov et al. [23] & $\begin{array}{l}\text { Ferumoxytol-DSC was more sensitive in detecting true progression than gadoteridol- } \\
\text { DSC }\end{array}$ \\
\hline \multicolumn{2}{|l|}{ DCE perfusion } \\
\hline \multicolumn{2}{|c|}{ Prognostic value for standard treatment (chemotherapy and radiation) } \\
\hline Cao et al. [17] & $\begin{array}{l}\text { Baseline as well as changes in rCBV were predictive of survival in patients with high- } \\
\text { grade gliomas }\end{array}$ \\
\hline \multicolumn{2}{|c|}{ Predictive value for response to antiangiogenic therapy } \\
\hline Sorensen et al. [10] & $\begin{array}{l}\text { Combining K-trans, microvessel volume, and circulating collagen IV was predictive of } \\
\text { overall and progression-free survival in patients with recurrent glioblastoma treated } \\
\text { with cediranib }\end{array}$ \\
\hline Cha et al. [12] & $\begin{array}{l}\text { Changes in rCBV were more strongly correlated with treatment response than } \\
\text { enhancing tumor size in recurrent malignant gliomas treated with thalidomide and } \\
\text { carboplatin }\end{array}$ \\
\hline
\end{tabular}


and $9 \%$ for patients with a maximum rCBV above 2.3. Even when controlling for astrocytoma grade, maximum rCBV remained prognostic.

Several studies have focused on using perfusion imaging to assess response to antiangiogenic therapy (ie, as a predictive rather than prognostic indicator). Sawlani et al. [9] studied 16 patients with recurrent GBM treated with bevacizumab and evaluated mean $\mathrm{rCBV}$, mean leakage coefficient, and hyperperfusion volume (HPV), which is defined as the fraction of tumor with an rCBV above a prespecified threshold, as potential biomarkers for treatment response. They found that the percent change in HPV (using an rCBV threshold of 1.00) from baseline to first follow-up had a statistically significant hazard ratio of 1.077 when correlated with time to progression. Although HPV may have potential as a predictive biomarker, the hazard ratio was only slightly greater than 1 , and the sample size was small; thus, additional refinements are needed to improve the clinical utility of this technique.

Sorensen et al. [10] studied 31 patients with recurrent GBM treated with cediranib, an inhibitor of the VEGF receptor tyrosine kinases. They calculated a "vascular normalization index" by combining K-trans, microvessel volume, and circulating collagen IV. A prior study that used a mouse model to evaluate the effect of cediranib found that edema alleviation was associated with a transient increase in circulating collagen IV, and it was thought that the increase in circulating collagen IV was reflective of basement membrane thinning [11]. The authors found that this new index (measured 1 day after treatment initiation) was predictive of overall and progression-free survival. The combination of imaging data with serum assays is a novel avenue of increasing the sensitivity and specificity of markers that may prove clinically useful.

Cha et al. [12] studied 18 patients with recurrent malignant gliomas treated with both thalidomide (an antiangiogenic agent) and carboplatin and found that changes in rCBV were more strongly correlated with treatment response based on clinical status than enhancing tumor size. In a multicenter trial with 36 patients with malignant gliomas treated with cilengitide (an angiogenesis inhibitor), Akella et al. [13] found that rCBF correlated with radiographic and clinical response. The correlation between $\mathrm{rCBV}$ and radiographic and clinical response approached significance. Both studies, as well as that from Sorensen et al. [10] discussed above, suggest that that perfusion imaging may be useful in the early assessment of response to angiogenesis inhibitors.

Perfusion imaging has also been investigated as a potential biomarker for prognosis following standard treatment (ie, without antiangiogenic therapy). In a retrospective study with 36 GBM patients treated with radiation and temozolomide, Mangla et al. [14] found that the percentage change in rCBV from the pre- and posttreatment measurements $(>5 \%$ or $\leq 5 \%$ ) was predictive of 1 -year survival with a sensitivity of $90 \%$ and a specificity of $60 \%$. Patients with increased $\mathrm{rCBV}$ had a median survival of 235 days, whereas those with decreased rCBV had a median survival of 529 days. In a larger study with 189 patients with low-grade and high-grade gliomas, Law et al. [15 • ] found that rCBV could be used to predict time to progression. Patients with rCBV less than 1.75 had a median time to progression of 3585 days, whereas patients with $\mathrm{rCBV}$ greater than 1.75 had a median time to progression of 265 days, indicating that survival in both high- and low-grade gliomas can be stratified by rCBV. For low-grade gliomas, increased $\mathrm{rCBV}$ may be a marker for degeneration into high-grade tumors, but Caseiras et al. [16] demonstrated that tumor growth on T1-weighted imaging is actually a stronger predictor of malignant transformation than change in $\mathrm{rCBV}$. Cao et al. [17] found that baseline as well as changes in $\mathrm{rCBV}$ were predictive of survival in 23 patients with high-grade gliomas. Thus, a number of studies have shown that high $\mathrm{rCBV}$ or increasing rCBV portends a poorer prognosis across multiple tumor grades. How much value this adds to standard MRI remains to be definitively determined.

The use of perfusion imaging to differentiate true progression from pseudoprogression has also been an area of interest. Barajas et al. [18••] investigated DSC parameters as a means of differentiating recurrent GBM from radiation necrosis (verified by histopathology) in 57 patients. They found that mean, maximum, and minimum relative peak height and $\mathrm{rCBV}$ were significantly greater in patients with recurrent tumor than in patients with radiation necrosis. In addition, mean, maximum, and minimum PSR values were significantly lower in patients with recurrent tumor than in those with radiation necrosis. However, there was substantial overlap in rCBV values, which presents challenges when applying a threshold to an individual patient. Hu et al. [19] examined the use of rCBV in differentiating high-grade glioma recurrence from posttreatment radiation effect in 13 patients. From these patients, they obtained 40 tissue specimens. Based on histopathology, 16 were identified as post-treatment radiation effect, and 24 were identified as recurrent glioma. In the post-treatment radiation effect group, rCBV values ranged from 0.21 to 0.71 . In the glioma recurrence group, rCBV values ranged from 0.55 to 4.64 . Hu et al. [19] found that an $\mathrm{rCBV}$ threshold value of 0.71 optimized the differentiation of the two groups. This threshold had a sensitivity of $91.7 \%$ and a specificity of $100 \%$. Sugahara et al. [20] also examined the ability of rCBV measurements to discriminate tumor recurrence from radiation treatment effect in mostly low- and high-grade gliomas, examining new enhancing lesions that developed within irradiated 
regions. rCBV ratios were greater than 2.6 in cases of recurrent tumor and less than 0.6 in cases of post-treatment radiation effect. Thus, differentiating tumors from radiation necrosis likely is dependent on typical rCBV values of various tumor types. Tumors with higher rCBV (eg, grade IV tumors and oligodendrogliomas [21]) may be more easily distinguishable from radiation necrosis than tumors with lower rCBV.

Although the reports from Barajas et al. [18••] and Hu et al. [19] both used DSC perfusion protocols, the ranges are lower for both tumor and radiation necrosis in the $\mathrm{Hu}$ et al. paper. Part of this difference may be due to the inclusion of both grade III and IV tumors in the report by $\mathrm{Hu}$ et al. [19], but it also is possible that differences in acquisition, processing, and MRI platforms may result in different thresholds across different sites even when using similar protocols. Variability in postprocessing methods can make comparisons across sites difficult [22]. Therefore, it will be important to establish standardized protocols to allow for more meaningful comparisons, particularly for the use in multicenter trials.

Most MR perfusion protocols use gadolinium-based contrast agents. However, Gahramanov et al. [23] argue that due to its tendency to extravasate into the extracellular space, gadolinium is a suboptimal contrast agent and may actually underestimate $\mathrm{rCBV}$ in some patients with progressive disease. They support the use of an iron oxide nanoparticle called ferumoxytol, which has chemical characteristics that are much different than gadolinium-based agents. It is thought that ferumoxytol, due to its larger molecular size, will exhibit significantly less extravasation from leaky vessels into the interstitium and thus allow for a more accurate measurement of rCBV. In their study, 14 GBM patients with new enhancing lesions after radiation therapy underwent DSC imaging with both gadoteridol and ferumoxytol. In all seven cases of clinically presumptive tumor recurrence, ferumoxytol-DSC revealed high rCBV values. In contrast, gadoteridol-DSC (a gadolinium-based agent) showed high rCBV values for only 4 of the 7 cases. Based upon these results, it appears that ferumoxytol-DSC is more sensitive in detecting true progression than gadoteridol-DSC. However, this small study relied upon clinical status and MRI changes to identify the enhancing lesion, and thus histopathologic confirmation of these findings would be desirable.

MR diffusion: functional diffusion maps and apparent diffusion coefficient histogram analysis

Diffusion-weighting imaging (DWI) is another MRI sequence under investigation in the assessment of gliomas. DWI is based on the movement of water molecules, and the values from these sequences are reported as the apparent diffusion coefficient (ADC). Lower ADC values reflect lower (more restricted) diffusion. Several physiologic properties of tumors may influence ADC values. Water molecules are generally more restricted in their movement within cells and less restricted in the extracellular space. Because necrosis involves degradation of cellular integrity, it is thought that necrosis increases ADC. In a similar way, edema increases interstitial fluid, thereby increasing ADC. Conversely, increased cellular density lowers ADC by restricting diffusion. Because of these relationships, DWI has been studied as a means of evaluating the effects of therapy on malignant gliomas.

The functional diffusion map (fDM) was developed to take advantage of the relationship between ADC and cell density by examining voxel-wise changes in ADC measured in the same patient over time [24-27]. By examining voxel-wise change in ADC, the sensitivity of detecting subtle changes in tumor cell density is dramatically increased. This technique has primarily been applied as a tool to predict response to cytotoxic chemotherapy and radiotherapy within the contrast-enhancing tumor bed [2426]; however, recent studies have demonstrated its utility outside regions of contrast enhancement [27-29] and as a tool for studying the effects of anti-VEGF therapy [30, 31]. Specifically, Ellingson et al. [31] found a significant decrease in ADC immediately following treatment with bevacizumab; however, the rate of change of tissue showing abnormally low ADC (fDM-classified "hypercellular" tissue) within regions of $\mathrm{T} 2$ signal abnormality was shown to be an early predictor of tumor progression, time to progression, and overall survival. In other words, if the fDM-classified hypercellular regions (low ADC) are expanding rapidly there is a higher likelihood of tumor recurrence and death, regardless of the specific treatment paradigm. Figure 1 demonstrates a patient with a recurrent GBM treated with bevacizumab and temozolomide illustrating substantial growth of hypercellular tumor regions prior to standard radiographic tumor progression.

Bevacizumab treatment has been noted to produce persistent diffusion restriction. Rieger et al. [32, 33] found that 13 out of 18 patients with recurrent malignant gliomas who received bevacizumab therapy exhibited diffusionrestricted areas, along with decreased ADC values. Areas of diffusion restriction had decreased rCBV. A biopsy of one of the diffusion-restricted areas revealed atypical necrosis with an upregulation of nuclear hypoxia-inducible factor- $1 \alpha$. These findings suggest that prolonged antiangiogenic therapy can result in chronic hypoxia, which, in turn, can lead to diffusion restriction. Gerstner et al. [34] presented a case report in which a patient with GBM experienced persistent diffusion restriction after bevacizumab treatment, and a biopsy revealed infiltrative tumor. Gerstner et al. [35] also observed areas of persistent diffusion restriction after the 


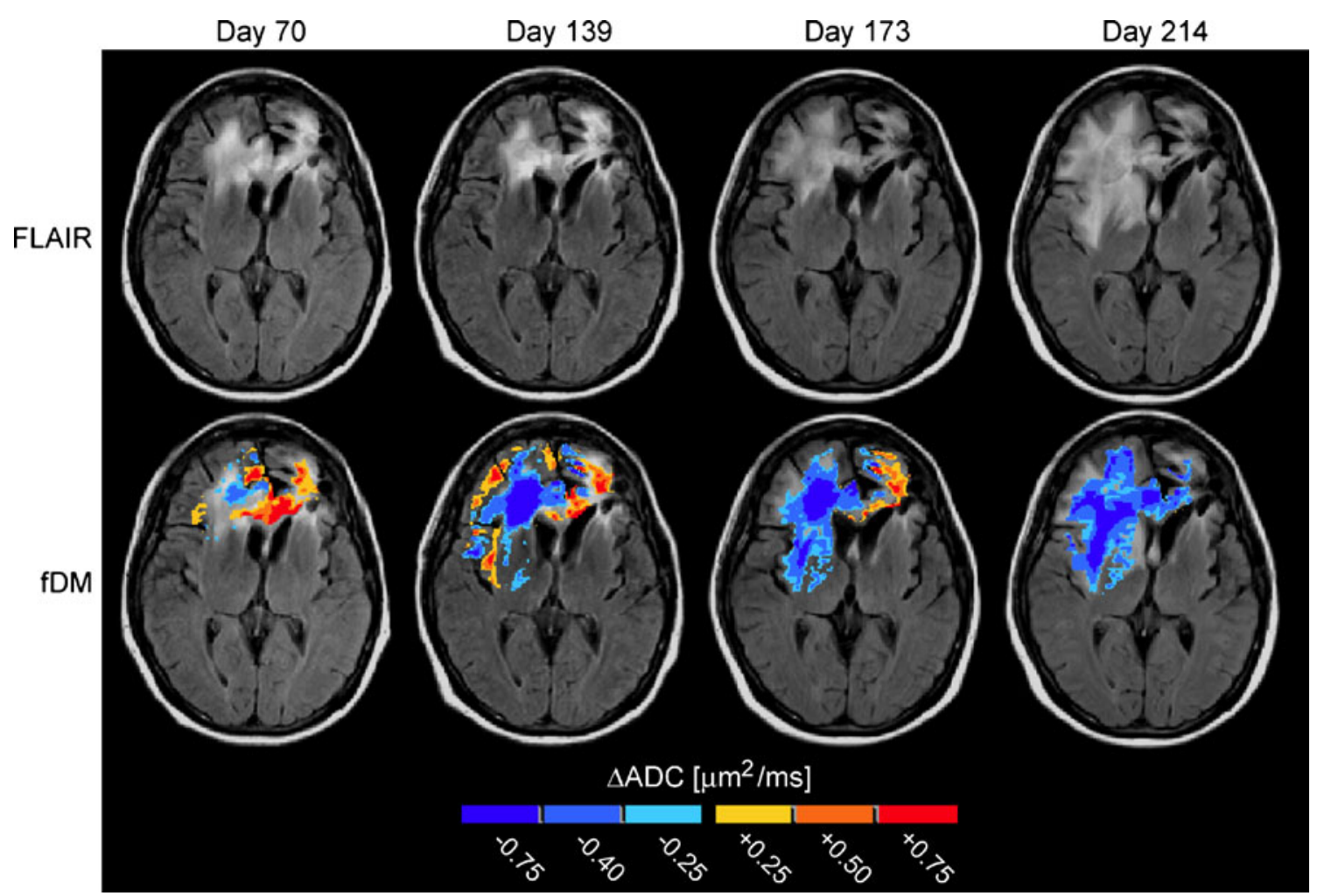

Fig. 1 Fluid-attenuated inversion recovery (FLAIR) images and functional diffusion maps (fDMs) in a patient with recurrent glioblastoma treated with bevacizumab and temozolomide. FLAIR images showing gradual spread of nonenhancing tumor (top row).

initiation of antiangiogenic therapy. They studied 30 patients with recurrent GBM treated with cediranib and found that this treatment resulted in lower ADC values. The percent volume of FLAIR hyperintensity comprised by low ADC increased significantly from baseline $(2.3 \%)$ to day 28 $(2.9 \%)$, day $56(5.0 \%)$, and day $112(6.3 \%)$ of cediranib therapy. They suggest that these findings are indicative of increasing infiltrative tumor. However, histopathologic correlation was not available. Because the studies by Gerstner et al. [34, 35] and Rieger et al. [32, 33] point to two different pathophysiologic processes that may explain persistent diffusion restriction, further investigation is needed to determine whether diffusion restriction is due to antiangiogenic effects of bevacizumab or whether it is due to tumor recurrence. Potentially, the degree of diffusion restriction could differentiate infiltrative tumor from hypoxic change.

Because ADC is influenced by cellular density, necrosis, and edema, ADC values can potentially be used as a noninvasive surrogate for VEGF expression and thus susceptibility to bevacizumab. We evaluated ADC histogram analysis as a predictive biomarker of response to bevacizumab in patients with recurrent GBM [36••]. Regions of enhancing tumor were segmented and mapped onto ADC images for ADC histogram generation. Tumors
fDMs showing increasing volume of low apparent diffusion coefficient (ADC) ("hypercellular") regions prior to radiographic failure (bottom row). Image days are with respect to pretreatment baseline (day 0$)$

with low ADC values prior to initiation of bevacizumab were more likely to progress by 6 months compared to those with high ADC values. This histogram analysis was $72.5 \%$ accurate in predicting 6-month progression-free survival, and it was more accurate than the Macdonald criteria at first follow-up. In the non-bevacizumab-treated cohort, ADC values were not predictive of median survival. If these findings are confirmed in a larger study, this would be the first validated MRI-based predictive biomarker for response to bevacizumab in patients with GBM.

\section{MR spectroscopy}

MR spectroscopy is a technique that allows for the characterization of the chemical makeup of a particular region of interest. Much of the research surrounding MR spectroscopy has focused on its potential ability to differentiate true progression from pseudoprogression. Smith et al. [37] studied 33 patients with primary intracranial neoplasms and found that ratios of brain metabolites can assist in differentiating recurrent tumor from post-treatment radiation effect. They found that patients with recurrent tumor had elevations in the choline (Cho)/creatine $(\mathrm{Cr}$ ) and $\mathrm{Cho} / \mathrm{N}$-acetylaspartate (NAA) ratios 
compared to those with post-treatment radiation effect. Patients with recurrent tumor also experienced a decrease in the $\mathrm{NAA} / \mathrm{Cr}$ ratio compared to those with posttreatment radiation effect. The Cho/NAA ratio had a sensitivity of $85 \%$ and a specificity of $69 \%$ in detecting true progression. In another study, Weybright et al. [38] examined 29 patients with new contrast-enhancing brain lesions in the vicinity of a previously treated brain neoplasm. Histopathology was used to confirm the identity of the lesion in 16 patients, and imaging and clinical follow-up were used to determine the identity of the lesion in the remaining 13 patients. Consistent with the study conducted by Smith et al. [37], Weybright et al. [38] found that the $\mathrm{Cho} / \mathrm{Cr}$ and Cho/NAA ratios were significantly greater in tumor recurrence compared with post-treatment radiation effect. The NAA/Cr ratio was significantly lower in recurrent tumor compared with post-treatment radiation effect. Some have suggested that creatine should not be used as a denominator in metabolite ratios because creatine may be anticorrelated with progression-free survival $[39,40]$. Although these studies were small, they suggest that MR spectroscopy may be helpful in differentiating true progression from pseudoprogression.

In addition to differentiating true progression from pseudoprogression, the Cho/NAA ratio may also be helpful as a biomarker for response to radiation therapy. $\mathrm{Oh}$ et al. [41] studied the predictive value of the Cho/ NAA ratio in 28 patients diagnosed with GBM after surgical resection but prior to radiation therapy. They found that patients with a large volume of metabolic abnormality (defined as a Cho/NAA ratio $>2$ ) had a significantly shorter survival time than those with a small volume of metabolic abnormality (12.0 and 17.1 months, respectively). Thus, the Cho/NAA ratio may have predictive value in assessing response to radiation.

\section{Radiotracers}

Radiotracers used in conjunction with positron emission tomography (PET) scans represent another group of potential biomarkers in assessing gliomas. By coupling radioactive tracers to molecules that are taken up by metabolically active cells, PET scans can detect and localize the presence of tumors and other cells with increased metabolism. A newer radiotracer is the nucleic acid analogue $18 \mathrm{~F}$-fluorothymidine $\left({ }^{18} \mathrm{~F}\right.$-FLT), which is used as an indicator of cellular proliferation. Chen et al. [42] studied 25 patients with gliomas and found that ${ }^{18} \mathrm{~F}$-FLT visualized all high-grade gliomas. In contrast, $18 \mathrm{~F}$ fluorodeoxyglucose $\left({ }^{18} \mathrm{~F}\right.$-FDG) did not detect tumor in five patients with recurrent glioma, demonstrating that ${ }^{18} \mathrm{~F}$-FLT had greater sensitivity than ${ }^{18} \mathrm{~F}-\mathrm{FDG}$. The lower sensitivity of ${ }^{18} \mathrm{~F}$-FDG-PET in this study may be due to the high background uptake of FDG in the cortex and other metabolically active regions, whereas many other available tracers, including FLT, have little or no uptake in normal brain. In this study, ${ }^{18} \mathrm{~F}$-FLT-PET was not only predictive of time to progression and survival, but it was also more strongly correlated with the Ki-67 staining (a histopathologic marker of proliferation that has been correlated with clinical outcomes) than ${ }^{18}$ F-FDG. In another study, Chen et al. [43] prospectively examined 19 patients with recurrent malignant gliomas treated with bevacizumab and irinotecan. ${ }^{18}$ F-FLT scans were performed before the initiation of treatment, after 1 to 2 weeks, and after 6 weeks; response was defined as a reduction in ${ }^{18} \mathrm{~F}$-FLT uptake by more than $25 \%$ after 6 weeks. Those who responded based on ${ }^{18} \mathrm{~F}$ FLT uptake survived three times as long as those who did not respond (10.8 vs 3.4 months). Both early and late ${ }^{18} \mathrm{~F}$ FLT responses were significant predictors of overall survival. These studies suggest that ${ }^{18}$ F-FLT has significant promise as a biomarker of outcome in patients with GBM, which can be acquired relatively early in the treatment cycle.

Amino acid tracers have also been studied. Potzi et al. [44] studied methyl- ${ }^{11} \mathrm{C}$-1-methionine $\left({ }^{11} \mathrm{C}\right.$-methionine $)$ as a means of assessing GBM in 28 patients. Although ${ }^{11} \mathrm{C}$ methionine uptake detected tumor in 24 out of 28 patients, there was no correlation between uptake and survival outcomes. Because it does not correlate with clinical outcomes, ${ }^{11} \mathrm{C}$-methionine may not be suitable as a response marker like ${ }^{18} \mathrm{~F}$-FLT, but may be more suitable for detecting disease recurrence, including nonenhancing tumor. For instance, Galldiks et al. [45] studied 12 patients with recurrent GBM and found that metabolically active tumor volume (defined as ${ }^{11} \mathrm{C}$-methionine uptake $\geq 1.3$ ) was significantly larger than the volume of gadolinium-enhanced tumor ( 30.17 vs $13.7 \mathrm{~cm}^{3}$ ). One drawback of ${ }^{11} \mathrm{C}$-methionine is its half-life of $20 \mathrm{~min}$. Due to its short half-life, ${ }^{11} \mathrm{C}$ methionine cannot be used in PET centers without a cyclotron, limiting its clinical utility. ${ }^{18} \mathrm{~F}$-labeled amino acid analogues with longer half-lives (eg, 3,4-dihydroxy-6-[18]F-fluoro-1phenylalanine $\left[{ }^{18} \mathrm{~F}\right.$-FDOPA $]$ ) have also been used to assess gliomas. Becherer et al. [46] studied 20 patients with supratentorial brain lesions (18 of which were primary brain tumors) and found that mean standardized uptake ratios were similar for ${ }^{18} \mathrm{~F}$-FDOPA and ${ }^{11} \mathrm{C}$-methionine (2.05 and 2.04 , respectively). Chen et al. [47] examined the diagnostic accuracy of ${ }^{18}$ F-FDOPA in assessing gliomas. They studied 81 patients with brain tumors and found that ${ }^{18} \mathrm{~F}$-FDOPA had a sensitivity of $98 \%$, a specificity of $86 \%$, a positive predictive value of $95 \%$, and a negative predictive value of $95 \%$. These studies indicate that amino acid tracers can reliably detect gliomas, and their ability to discriminate gliosis from nonenhancing tumor merits additional investigation. 


\section{Conclusions}

The search for clinically useful biomarkers for treatment response in malignant gliomas has been challenging. Recently, attempts to assess gliomas using imaging have broadened in focus from strictly anatomic measurements to evaluating the utility of MRI techniques that quantify changes in tumor physiology. Based on preliminary studies, new physiologic imaging techniques may have the ability to detect drug susceptibility or resistance prior to changes in tumor size. If these efforts are successful, this could allow for earlier treatment decisions and more individually tailored medicine. Unfortunately, the clinical utility of these physiologic imaging techniques remains unproven and the methods unstandardized. To date, no perfusion-based imaging biomarkers have been validated in large trials. ADC histogram analysis has been preliminarily shown to have predictive value in predicting response to bevacizumab. Confirmation in a larger study is ongoing. As advances occur in the development of therapies that target specific biochemical pathways and alter tumor physiology in potentially predictable ways, the validation of physiologic biomarkers will become increasingly important.

Disclosure Conflicts of interest: W.B. Pope: has received honoraria as a consultant for Genentech; J.R. Young: none; B.M. Ellingson: none.

Open Access This article is distributed under the terms of the Creative Commons Attribution Noncommercial License which permits any noncommercial use, distribution, and reproduction in any medium, provided the original author(s) and source are credited.

\section{References}

Papers of particular interest, published recently, have been highlighted as:

•- Of major importance

1. Norden AD, Drappatz J, Wen PY: Novel anti-angiogenic therapies for malignant gliomas. Lancet Neurol. Dec 2008;7(12):11521160.

2. Wen PY, Macdonald DR, Reardon DA, et al.: Updated response assessment criteria for high-grade gliomas: response assessment in neuro-oncology working group. J Clin Oncol. Apr 10 2010;28 (11):1963-1972.

3. Rees JH, Smirniotopoulos JG, Jones RV, Wong K: Glioblastoma multiforme: radiologic-pathologic correlation. Radiographics. Nov 1996;16(6):1413-1438; quiz 1462-1413.

4. Clarke JL, Chang S: Pseudoprogression and pseudoresponse: challenges in brain tumor imaging. Curr Neurol Neurosci Rep. May 2009;9(3):241-246.
5. Brandsma D, van den Bent MJ: Pseudoprogression and pseudoresponse in the treatment of gliomas. Curr Opin Neurol. December 2009;22(6):633-638.

6. Kim JH, Chung YG, Kim CY, et al.: Upregulation of VEGF and FGF2 in normal rat brain after experimental intraoperative radiation therapy. J Korean Med Sci. Dec 2004;19(6):879-886.

7. Gonzalez J, Kumar AJ, Conrad CA, Levin VA: Effect of bevacizumab on radiation necrosis of the brain. Int $\mathrm{J}$ Radiat Oncol Biol Phys. Feb 1 2007;67(2):323-326.

8. Hirai T, Murakami R, Nakamura $H$, et al.: Prognostic value of perfusion MR imaging of high-grade astrocytomas: long-term follow-up study. AJNR Am J Neuroradiol. Sep 2008;29(8):15051510.

9. Sawlani RN, Raizer J, Horowitz SW, et al.: Glioblastoma: a method for predicting response to antiangiogenic chemotherapy by using MR perfusion imaging-pilot study. Radiology. May 2010;255(2):622-628.

10. Sorensen AG, Batchelor TT, Zhang WT, et al.: A "vascular normalization index" as potential mechanistic biomarker to predict survival after a single dose of cediranib in recurrent glioblastoma patients. Cancer Res. Jul 1 2009;69(13):5296-5300.

11. Kamoun WS, Ley CD, Farrar CT, et al.: Edema control by cediranib, a vascular endothelial growth factor receptor-targeted kinase inhibitor, prolongs survival despite persistent brain tumor growth in mice. J Clin Oncol. May 20 2009;27(15):25422552.

12. Cha S, Knopp EA, Johnson G, et al.: Dynamic contrast-enhanced T2-weighted MR imaging of recurrent malignant gliomas treated with thalidomide and carboplatin. AJNR Am J Neuroradiol. May 2000;21(5):881-890.

13. Akella NS, Twieg DB, Mikkelsen T, et al.: Assessment of brain tumor angiogenesis inhibitors using perfusion magnetic resonance imaging: quality and analysis results of a phase I trial. J Magn Reson Imaging. Dec 2004;20(6):913-922.

14. Mangla R, Singh G, Ziegelitz D, et al.: Changes in relative cerebral blood volume 1 month after radiation-temozolomide therapy can help predict overall survival in patients with glioblastoma. Radiology. Aug 2010;256(2):575-584.

15. •- Law M, Young RJ, Babb JS, et al.: Gliomas: predicting time to progression or survival with cerebral blood volume measurements at dynamic susceptibility-weighted contrast-enhanced perfusion MR imaging. Radiology. May 2008;247(2):490-498. This large, well-designed study demonstrated that $r C B V$ can predict time to progression in both high-grade and low-grade gliomas.

16. Caseiras GB, Ciccarelli O, Altmann DR, et al.: Low-grade gliomas: six-month tumor growth predicts patient outcome better than admission tumor volume, relative cerebral blood volume, and apparent diffusion coefficient. Radiology. Nov 2009;253(2):505-512.

17. Cao Y, Tsien CI, Nagesh V, et al.: Survival prediction in highgrade gliomas by MRI perfusion before and during early stage of RT [corrected]. Int J Radiat Oncol Biol Phys. Mar 1 2006;64 (3):876-885.

18. •• Barajas RF, Jr., Chang JS, Segal MR, et al.: Differentiation of recurrent glioblastoma multiforme from radiation necrosis after external beam radiation therapy with dynamic susceptibilityweighted contrast-enhanced perfusion MR imaging. Radiology. Nov 2009;253(2):486-496. This well-conducted study demonstrated that DSC parameters ( $r C B V$, relative peak height, and PSR) may help differentiate recurrent glioblastoma from radiation necrosis.

19. Hu LS, Baxter LC, Smith KA, et al.: Relative cerebral blood volume values to differentiate high-grade glioma recurrence from posttreatment radiation effect: direct correlation between image-guided tissue histopathology and localized dynamic susceptibility-weighted contrast-enhanced perfusion MR imaging measurements. AJNR Am J Neuroradiol. Mar 2009;30(3):552-558. 
20. Sugahara T, Korogi Y, Tomiguchi S, et al.: Posttherapeutic intraaxial brain tumor: the value of perfusion-sensitive contrastenhanced MR imaging for differentiating tumor recurrence from nonneoplastic contrast-enhancing tissue. AJNR Am J Neuroradiol. May 2000;21(5):901-909.

21. Lev MH, Ozsunar Y, Henson JW, et al.: Glial tumor grading and outcome prediction using dynamic spin-echo MR susceptibility mapping compared with conventional contrast-enhanced MR: confounding effect of elevated rCBV of oligodendrogliomas [corrected]. AJNR Am J Neuroradiol. Feb 2004;25(2):214-221.

22. Paulson ES, Schmainda KM: Comparison of dynamic susceptibility-weighted contrast-enhanced MR methods: recommendations for measuring relative cerebral blood volume in brain tumors. Radiology. Nov 2008;249(2):601-613.

23. Gahramanov S, Raslan AM, Muldoon LL, et al.: Potential for Differentiation of Pseudoprogression from True Tumor Progression with Dynamic Susceptibility-weighted Contrast-enhanced Magnetic Resonance Imaging using Ferumoxytol vs. Gadoteridol: A Pilot Study. Int J Radiat Oncol Biol Phys. Apr 132010.

24. Moffat BA, Chenevert TL, Meyer CR, et al.: The functional diffusion map: An imaging biomarker for the early prediction of cancer treatment outcome. Neoplasia. 2006;8(4):259-267.

25. Hamstra DA, Chenevert TL, Moffat BA, et al.: Evaluation of the functional diffusion map as an early biomarker of time-toprogression and overall survival in high-grade glioma. Proc Nat Acad Sci. 2005;102(46):16759-16764.

26. Hamstra DA, Galbán CJ, Meyer CR, et al.: Functional diffusion map as an early imaging biomarker for high-grade glioma: correlation with conventional radiologic response and overall survival. J Clin Oncol. 2008;26(10):3387-3394.

27. Ellingson BM, Malkin MG, Rand SD, et al.: Validation of functional diffusion maps (fDMs) as a biomarker for human glioma cellularity. J Magn Reson Imaging. 2010;31(3):538-548.

28. Ellingson BM, Malkin MG, Rand SD, et al.: Functional diffusion maps applied to FLAIR abnormal areas are valuable for the clinical monitoring of recurrent brain tumors. Proc Intl Soc Mag Reson Med. 2009;17:285.

29. Ellingson BM, Rand SD, Malkin MG, Schmainda KM: Utility of functional diffusion maps to monitor a patient diagnosed with gliomatosis cerebri. J Neurooncol. 2010;97(3):419-423.

30. Ellingson BM, Malkin MG, Rand SD, et al.: Comparison of cytotoxic and anti-angiogenic treatment responses using functional diffusion maps in FLAIR abnormal regions. Proc Intl Soc Mag Reson Med. 2009;17:1010.

31. Ellingson BM, Malkin MG, Rand SD, et al.: Volumetric analysis of functional diffusion maps (fDMs) is a predictive imaging biomarker for cytotoxic and anti-angiogenic treatments in malignant glioma. J Neurooncol. 2010; In Press.

32. Rieger $\mathrm{J}$, Bahr $\mathrm{O}$, Muller $\mathrm{K}$, et al.: Bevacizumab-induced diffusion-restricted lesions in malignant glioma patients. J Neurooncol. Aug 2010;99(1):49-56.

33. Rieger J, Bahr O, Ronellenfitsch MW, et al.: Bevacizumab-Induced Diffusion Restriction in Patients With Glioma: Tumor Progression or Surrogate Marker of Hypoxia? J Clin Oncol. Jun 282010.
34. Gerstner ER, Frosch MP, Batchelor TT: Diffusion magnetic resonance imaging detects pathologically confirmed, nonenhancing tumor progression in a patient with recurrent glioblastoma receiving bevacizumab. J Clin Oncol. Feb 20 2010;28(6):e91-93.

35. Gerstner ER, Chen PJ, Wen PY, et al.: Infiltrative patterns of glioblastoma spread detected via diffusion MRI after treatment with cediranib. Neuro Oncol. May 2010;12(5):466-472.

36. •- Pope WB, Kim HJ, Huo J, et al.: Recurrent glioblastoma multiforme: ADC histogram analysis predicts response to bevacizumab treatment. Radiology. Jul 2009;252(1):182-189. This well-conducted study demonstrated that ADC histogram analysis is $72.5 \%$ accurate in predicting 6-month progression-free survival in patients with glioblastoma treated with bevacizumab.

37. Smith EA, Carlos RC, Junck LR, et al.: Developing a clinical decision model: MR spectroscopy to differentiate between recurrent tumor and radiation change in patients with new contrast-enhancing lesions. AJR Am J Roentgenol. Feb 2009;192(2):W45-52.

38. Weybright P, Sundgren PC, Maly P, et al.: Differentiation between brain tumor recurrence and radiation injury using MR spectroscopy. AJR Am J Roentgenol. Dec 2005;185(6):1471-1476.

39. Hattingen E, Delic O, Franz K, et al.: (1)H MRSI and progressionfree survival in patients with WHO grades II and III gliomas. Neurol Res. Jul 2010;32(6):593-602.

40. Hattingen E, Raab P, Franz K, et al.: Prognostic value of choline and creatine in WHO grade II gliomas. Neuroradiology. Sep 2008;50(9):759-767.

41. Oh J, Henry RG, Pirzkall A, et al.: Survival analysis in patients with glioblastoma multiforme: predictive value of choline-to-Nacetylaspartate index, apparent diffusion coefficient, and relative cerebral blood volume. J Magn Reson Imaging. May 2004;19 (5):546-554.

42. Chen W, Cloughesy $\mathrm{T}$, Kamdar N, et al.: Imaging proliferation in brain tumors with 18 F-FLT PET: comparison with 18 F-FDG. J Nucl Med. Jun 2005;46(6):945-952.

43. Chen W, Delaloye S, Silverman DH, et al.: Predicting treatment response of malignant gliomas to bevacizumab and irinotecan by imaging proliferation with $[18 \mathrm{~F}]$ fluorothymidine positron emission tomography: a pilot study. J Clin Oncol. Oct 20 2007;25(30):4714-4721.

44. Potzi C, Becherer A, Marosi C, et al.: $[11 \mathrm{C}]$ methionine and [18 F] fluorodeoxyglucose PET in the follow-up of glioblastoma multiforme. J Neurooncol. Sep 2007;84(3):305-314.

45. Galldiks N, Ullrich R, Schroeter M, et al.: Volumetry of [(11)C]methionine PET uptake and MRI contrast enhancement in patients with recurrent glioblastoma multiforme. Eur J Nucl Med Mol Imaging. Jan 2010;37(1):84-92.

46. Becherer A, Karanikas G, Szabo M, et al.: Brain tumour imaging with PET: a comparison between [18 F]fluorodopa and [11 C]methionine. Eur J Nucl Med Mol Imaging. Nov 2003;30(11):1561-1567.

47. Chen W, Silverman DH, Delaloye S, et al.: 18 F-FDOPA PET imaging of brain tumors: comparison study with 18 F-FDG PET and evaluation of diagnostic accuracy. J Nucl Med. Jun 2006;47 (6):904-911. 